THE INTERACTIONS BETWEEN MUNICIPAL SOCIOECONOMIC STATUS AND AGE ON HIP FRACTURE RISK

\title{
Carla Maria Oliveira
}

Rua do Campo Alegre, 823, 4150-180 Porto, INEB, Instituto de Engenharia Biomédica, Portugal

Rua das Taipas 135, 4050-600, Porto, ISPUP, Instituto de Saúde Pública da Universidade do Porto,

Portugal

Al. Prof. Hernâni Monteiro, 4200 - 319 Porto, Departamento de Epidemiologia Clínica, Medicina Preditiva e Saúde Pública, Faculdade de Medicina da Universidade do Porto, Portugal

carlaoliveir@gmail.com

Theodoros Economou

College of Engineering, Mathematics and Physical Sciences, University of Exeter, North Park Road, EX4 4QF, Exeter, UK.

t.economou@ex.ac.uk

Trevor Bailey

College of Engineering, Mathematics and Physical Sciences, University of Exeter, North Park Road, EX4 4QF, Exeter, UK.

t.c.bailey@ex.ac.uk

Denisa Mendonça

Rua das Taipas 135, 4050-600, Porto, ISPUP, Instituto de Saúde Pública da Universidade do Porto, Portugal

Rua de Jorge Viterbo Ferreira n.ㅇ 228, 4050-313 Porto, ICBAS, Instituto de Ciências Biomédicas Abel Salazar da Universidade do Porto

dvmendon@icbas.up.pt

Maria Fátima Pina

Al. Prof. Hernâni Monteiro, 4200 - 319 Porto, Departamento de Epidemiologia Clínica, Medicina Preditiva e Saúde Pública, Faculdade de Medicina da Universidade do Porto, Portugal Rua do Campo Alegre, 823, 4150-180 Porto, INEB, Instituto de Engenharia Biomédica, Portugal Rua das Taipas 135, 4050-600, Porto, ISPUP, Instituto de Saúde Pública da Universidade do Porto, Portugal

fpina@med.up.pt 
Corresponding author: C Oliveira; carlaoliveir@gmail.com; phone +351966060193; Fax: 351 226094567; Rua do Campo Alegre, 823, 4150-180 Porto, INEB, Instituto de Engenharia Biomédica, Portugal

Acknowledgements:

This work was supported by FEDER funds through the Programa Operacional Factores de Competitividade (COMPETE) and by Portuguese funds through Fundação para a Ciência e a Tecnologia (FCT) within the framework of the project PTDC/SAU-EPI/113424/2009 grant.

We also acknowledge the Central Administration of Health Services (ACSS) for the data from the National Hospital Discharge Register.

Conflict of Interest:

Carla Maria Oliveira, Theodoros Economou, Trevor Bailey, Denisa Mendonça and Maria Fátima Pina declare that they have no conflict of interest.

Role of funding source:

The funder Fundação para a Ciência e Tecnologia - FCT has no role in this paper. 


\section{Mini-abstract}

Age modifies the effect of area-level socioeconomic status (SES) in the risk of fragility hip fractures (HF). For older individuals risk of HF increases as SES increases. For younger, risk of HF increases as SES decreases. Our study may aid decisions makers and medical guidelines for HF prevention. 
Background: The effect of socioeconomic status (SES) on hip fracture (HF) incidence remains unclear.

Objective: To evaluate the association between HF incidence and municipality-level SES as well as interactions between age and SES.

Methods: From the Portuguese Hospital Discharge Database we selected hospitalizations (2000-2010) of patients aged 50+, with HF diagnosis (codes 820.x, ICD9.CM), caused by traumas of low/moderate energy, excluding bone cancer cases and readmissions for after-care. Municipalities were classified according to SES (deprived to affluent) using 2001 Census data. A spatial Bayesian hierarchical regression model (controlling for data heterogeneity effect and spatial autocorrelation), using the Poisson distribution, was used to quantify the Relative Risk (RR) of $\mathrm{HF}, 95 \%$ credible interval $(95 \% \mathrm{Crl})$, and to analyze the interaction between age and SES, after adjusting for rural conditions.

Results: There were $96,905 \mathrm{HF}, 77.3 \%$ of which were on women who on average were older than men (mean age $81.2 \pm 8.5$ vs $78.2 \pm 10.1$ years) at admission $(p<0.001)$. In women, there was a lower risk associated with better SES: RR=0.83 (95\% Crl 0.65-1.00) for affluent versus deprived. There was an inverse association between SES and HF incidence rate in the youngest and a direct association in the oldest, for both sexes, but significant only between deprived and affluent in older ages ( $\geq 75 y e a r s)$.

Conclusions: Interaction between SES and age may be due to inequalities in lifestyles, access to health systems and preventive actions. These results may help decision-makers to better understand the epidemiology of hip fractures and to better direct the available funding.

Keywords: osteoporosis, hip fractures, socioeconomic status, spatial epidemiology, interaction 


\section{Introduction}

Bone fractures in the elderly[1] are mainly caused by a combination of low bone mineral density (BMD) in individuals with osteoporosis, and traumas of low/moderate energy after a fall from standing height or less, and they are more frequent among women [2]. From all such fragility fractures, hip fractures (HF) are the most severe, because of the high costs associated with treatment and recovery [3] and the high physical disability, social dependence and mortality among the elders after a fracture [2, 4].

In Europe, incidence rates of HF are seven times higher in the northern countries when compared to the southern countries [5], although when analyzing the incidence of HF at municipality level, there are strong geographical differences within the countries - in Portugal incidence rates of HF in the northeast and south of the country are similar to those reported in Finland [6]. The reasons for such geographical differences are not well understood but a plausible explanation is that such differences are associated with socioeconomic and environmental contexts [7]: geographical variations in diet, body height and weight, smoking, alcohol intake, physical activity and uptake of anti-osteoporosis medication [2] can be associated with HF incidence and may explain the geographical differences in the incidence rates. Heterogeneity in the policy framework, service provision and service uptake for osteoporotic fractures may be also a reason for the difference in HF risk between regions[8]. In addition, unhealthy lifestyles and exposure to environmental risk factors tend to be higher in deprived areas [9].

Unfortunately, relatively little work has been published regarding the association between HF risks and socioeconomic status (SES), or some proxy:

Studies consistently showed a significant association with marital status (a decrease in those married or living with someone) [10-12], occupational category (reduced risk for employed vs unemployed and for tradespeople vs laborers) $[13,14]$ and residence type (living in large vs small homes decreases the risk) [11]. A study in Stockholm $[10,11,15]$ indicated reduced risk for those with medium or high incomes compared to low incomes. For Australia [16], there was reduced risk for those with private health insurance compared to ones without. In the Trend region (UK) study, an increased risk of falls in areas of lower SES (for adults aged 75 years or over) was described but without significant association with $\mathrm{HF}$ [7]. Nevertheless, some inconsistencies do exists, for example the role of income or education in the risk of HF: there were different associations in different age groups, which might suggest an age interaction with SES [17].

The age modifiable effect on the association between SES and HF is not well studied in the current literature. A nationwide case-control study in Denmark described a reduction in the risk of any type of fracture in younger age groups ( $<40$ years old) among those with higher education levels, while the opposite was observed among subjects aged 60 years or more: higher risk in the higher education levels [18]. In Wales, those over 74 years-old living in affluent neighborhoods have greater risk compared with those living in more deprived areas, while individuals below 74 living in more deprived areas, had an increased risk of HF when compared with those living in more affluent areas $[15,19]$. In the Stockolm 
metropolitan area, a higher risk of $\mathrm{HF}$ was found in the elderly living in more affluent areas, corroborating these findings. [18]

Better understanding of the relationship between $\mathrm{HF}$ and socioeconomic and environmental factors, and their interactions with age, can help in better targeting prevention measures of HF [7]. However, there is a gap in the scientific literature regarding this issue. Our objective here is to investigate and quantify the relationship between HF and SES as well as the relevance of the interaction between age and SES on HF incidence, in Portugal using data in the period 2000-2010. To this end, we propose a flexible statistical model, which accounts for spatial heterogeneity in the data while also allowing for unobserved but also observed factors in the form of predictors.

\section{Materials and Methods:}

\section{Study area}

The study area is Continental Portugal, which had 10,057,999 inhabitants in 2010, distributed heterogeneously throughout 278 municipalities, with a median of 15,741 inhabitants per municipality. The least populated municipality has 1,836 inhabitants and the most populated is the capital, Lisbon, with 548,422 inhabitants. The population over the age of 50 rose from 3,298,900 (in 2000) to 3,789,091 (in 2010)[20]. In Portugal, although it is a developed country, inequality of wealth distribution, measured through the GINI index, is among the highest in the European region [21].

\section{Data}

We used data from the National Hospital Discharge Register (NHDR), which has been mandatory for all Portuguese public hospitals since 1997 and whose quality is assessed regularly by internal (hospitals) and external (ACSS - Central Administration of the National System) auditors [22]. Each record in the NHDR corresponds to one discharge and contains information such as: sex; age; first cause of admission and main diagnosis (and up to 19 secondary causes and 19 secondary diagnoses), coded according to the International Classification of Diseases, version 9, Clinical Modification (ICD9-CM); municipality of patient's residence; date of admission and discharge, hospital to and hospital from whenever there is a transfer between hospitals, among others [23]. In Portugal, access to the national health-care system is universal and tendentiously free-of-charge: contributions are based upon citizens' social and economic conditions [22]. All the patients with a HF are hospitalized for treatment and due to the high costs involved, HF are primarily treated in public hospitals. Therefore, the admissions by HF registered in the NHDR represent almost the totality nationwide and can be seen as a proxy of incidence. For reasons of confidentiality we did not have access to patient's code and therefore recurrent fractures in the same patient could not be identified.

We selected all hospital admissions, from 1 January 2000 to 31 December 2010, of patients aged 50 years and over, with a discharge diagnosis of HF (ICD9-CM codes 820.x) caused by traumas of 
low/moderate energy (ICD9-CM codes E849.0, E849.7 and E880-E888). We excluded readmissions for after care (ICD9-CM codes 996.4 and V54.x) and pathological fractures (ICD9-CM codes 170.x and 171.x). No such data was available for the two autonomous regions, the archipelagos of the Azores or Madeira (representing $5 \%$ of the Portuguese population), and therefore they were not included in the study. Counts of HF were stratified by municipality of patient's residence, admission year, sex and 5-year age groups $(50-54 \ldots 80-84,85+)$.

To calculate the population at-risk or person-years we aggregated population per municipality, sex and 5-year age groups using data from the 2001 Census and from the annual official estimates for all the other years (INE - Statistics Portugal [20]).

The socioeconomic characterization of municipalities was based on data from the last available Census 2001 (INE - Statistics Portugal [20]). In Portugal there were no significant changes in SES during the study period and therefore the relative position in the SES rank of municipalities between 2000 and 2010 remains stable (results not shown); each municipality was characterized by a set of variables related to buildings, households, families and individuals. The variables included were: proportion of population by age groups and sex; proportion of retired individuals (by sex); proportion of widows; proportion of individuals receiving social support; proportion of illiteracy; ageing and youth dependency indexes; proportion of individuals living alone; mean number of rooms per household; mean number of individuals per household; unemployment rate; proportion of subjects with higher and basic education; proportion of subjects by category of occupation (managers/professionals/technicians; services/sales workers; skilled agricultural/plant \& machine operators); income; proportion of residences and buildings with/without public water supply, mains or otherwise; and proportion of households with heating, by type of heating. To create the SES index, we performed a principal component analysis to reduce the set of variables described above to four components, which explained $75.8 \%$ of the total variability. Afterwards, we used the four components to develop a hierarchical cluster analysis [24], using the Ward's method. We used three clusters of SES to analyze the association with HP incidence: affluent, medium and deprived (Figure 1). The affluent SES category aggregates municipalities with younger population, higher educational level, higher percentage of employed individuals and good housing conditions (plumbing, heating and bathroom facilities and shower). The medium SES category aggregates municipalities with population older than in affluent group, higher illiteracy rate, low indices per capita, higher percentage of individuals employed in agriculture, forestry and industry. The deprived SES category aggregates municipalities with the highest percentage of elderly, the highest illiteracy rate, the highest rate of people living alone, the lowest level of education, the lowest indices per capita, higher percentage of individuals with rural activities, the highest percentage of house with no running water and no bathroom facilities and shower and the highest percentage of individuals receiving unemployment benefits.

The distribution of the population in Continental Portugal significantly differ per SES group $(p<0.001)$. The median population per municipality increases with SES: 1,607 inhabitants (interquartile rage (IQR) 
1,207 - 2,402) in the deprived SES cluster; 3,473 inhabitants (IQR 2,386 - 6,285) in the medium SES cluster and 7,426 inhabitants (IQR 3,453-12,706) in the affluent SES cluster. [20].

We considered rural conditions as a possible confounder of the association between SES and HF incidence rate, since rural areas can be associated with a lower risk of $\mathrm{HF}[25]$ and some rural regions may have lower SES. Therefore we classified the municipalities as rural, urban and semi-urban (Figure 1) based on the official information available [20].

\section{Statistical analysis}

For individual's characteristics, differences in mean age by sex and SES were analyzed using ANOVA and the Tukey HSD test for multiple comparisons; statistical significance level (two-sided) was set at 5\%.

To assess the relationship between mean HF rates and SES, a spatial Bayesian hierarchical regression model was implemented. Age and rural conditions were included in the model as predictors to allow for the (possible) different behavior of HF rate between various categories of age and rural conditions. An interaction term between age and SES was also included to capture the possible modifiable effect of age in the relation between SES and HF incidence. The interaction term in our model is essentially another categorical variable with $\mathrm{N}$ levels, where $\mathrm{N}$ is the number of age groups times the number of SES groups. All analyses were performed separately for each sex.

We assumed that the number of HF, NFrat ${ }_{i j}$, in a specific age group $i(i=1, \ldots, 8)$ and in a specific municipality $j(j=1, \ldots, 278)$ is distributed as a Poisson random variable with mean $\lambda_{i j}=N P o p_{i j} \varrho_{i j}$ where $\mathrm{NPop}_{i j}$ is the population in age group $i$ and municipality $j$, and $\varrho_{i j}$ is the incidence rate of HF per unit population in age group $i$ and municipality $j$; so that, for instance, the quantity $\varrho_{i j} / \varrho_{k j}$ is the relative risk (RR) of age group $i$ to reference age group $k(k=1, \ldots, 8)$. For each sex, the parameters of interest were estimate by the following model:

$\operatorname{NFrat}_{i j} \mid\left(\psi_{j}, \phi_{j}\right) \sim \operatorname{Pois}\left(\lambda_{i j}\right)$

$\log \left(\lambda_{i j}\right)=\log \left(N P o p_{i j}\right)+\log \left(\varrho_{i j}\right)=\log \left(N P o p_{i j}\right)+\beta_{0}+\beta_{1} A g G r_{i}+\beta_{2} S E S_{j}+\beta_{3} R u r U r b_{j}$

$+\beta_{12} A g G r_{i} * S E S_{j}+\psi_{j}+\phi_{j}$

$\psi_{j} \sim N\left(0, \sigma_{\psi}^{2}\right)$

$\phi_{j} \mid \phi_{(-j)} \sim N\left(\bar{\phi}_{j}, \sigma_{\phi}^{2} / m_{j}\right) ; \phi_{(-j)}=\left\{\phi_{k}: \mathrm{k} \neq \mathrm{j} \wedge \mathrm{k} \in A_{j}\right\}$

where $A_{j}$ is the set of neighbors of municipality $j$; the $A g G r_{i}$ is the age group $i$; the SES $\left(S E S_{j}\right)$ and the rural condition $\left(\operatorname{RurUrb}_{j}\right)$ are characteristics of the municipality $j ; \psi_{j}$ is the non-spatial random effect and $\phi_{j}$ is the spatially structured random effect $\left(\bar{\phi}_{j}\right.$ is the average of the $\phi_{k}$ that are adjacent to $\phi_{j}$ and $m_{j}$ is the number of these adjacencies). The joint model for the $\phi_{j}$ 's is the so called conditional autoregressive (CAR) normal prior [26]. With this model we attempt to modeling the effect of AGE, SES and the interaction AGE*SES (adjusting for rurality) on the risk of hip fracture incidence rates where $\beta_{1}$, $\beta_{2}$ and $\beta_{12}$ are the age, sex and interaction term coefficients, respectively.

The model included two random effects (or latent variables) at the municipality level. One of the random effects $\left(\psi_{j}\right)$ accounted for the unexplained heterogeneity in HF rate due to unobserved municipality-level factors while the other $\left(\phi_{j}\right)$ allowed for spatially structured dependence in 
measurements of HF in municipalities that were spatially close. The latter relates to the fact that HF incidence in two nearby municipalities tends to be more similar (in terms of risk) than two areas chosen randomly.

The model described above was implemented in a Bayesian framework and estimated using Markov Chain Monte Carlo (MCMC). In this framework, parameters are treated as random variables whose "prior" distribution expresses the uncertainty about their value before any data is observed. After data is obtained though, prior distributions (or simply priors), are combined with the data through Bayes theorem to produce the posterior distributions (or simply the posteriors) of each parameter. The posteriors express the uncertainty about model parameters after data is observed and all statistical inference is based solely on the posteriors. MCMC is a numerical technique which produces samples of values that eventually converge (after a certain "burn-in" number) to samples of values from the posterior (distribution) of each parameter.

Uninformative prior distributions were assumed for the model parameters. Parameters $\beta_{0}, \beta_{1}, \beta_{2}, \beta_{3}, \beta_{12}$ were given Gaussian priors with zero mean and large variance (1000) whereas $\sigma_{\psi}^{2}$ and $\sigma_{\phi}^{2}$ priors were assumed to be gamma distributed: gamma $(0.5,0.0005)$.

All the analyses were performed with the Statistical software R version 2.14.1 (Project for Statistical Computing)[27] and WinBUGS14 (WinBUGS14, Cambridge, UK)[28] using R2WinBUGS package to connect both tools. WinBUGS uses Gibbs sampling, a particular MCMC technique, to produce samples from the posterior distribution of each parameter (or simply posterior samples).

The RR and its $95 \%$ credible interval $(95 \% \mathrm{Crl})$ were estimated using the sample mean and the $2.5 \%$ and 97.5\% empirical quantiles of the posterior samples from each parameter of interest. These samples are based on two MCMC chains with 100,000 iterations each and a burn-in period of 10,000.

\section{Results:}

There were 98,186 admissions for HF in Continental Portugal between 2000 and 2010. From those we excluded 585 because of missing data for municipality of residence and 696 because of readmissions for after care; our final sample includes 96,905 fractures of which 74,928 (77.3\%) were in women. On average, women were older than men at admission $(p<0.001)$, with a mean age (Standard Deviation SD) of 81.2 (8.5) versus 78.2 (10.1) years old, and the same pattern was observed in all the SES; the highest age difference (3.3 years $95 \% \mathrm{Cl}$ : 3.0-3.5) between sexes was in the affluent municipalities and the lowest (2.5 years $95 \% \mathrm{Cl}: 2.0-3.1$ ) was in the deprived municipalities (Table 1 ).

Age differences between all SES were observed in both sexes, except between affluent and medium SES in women. Men and women were older at admission in the deprived SES and younger in the affluent SES; such differences in the mean age of admission between SES were higher in men (1.8 years $95 \% \mathrm{Cl}$ : 1.2-2.3) than in women (1.0 years $(95 \% \mathrm{Cl}: 0.7-1.3)$ (Table 1$)$.

Figure 1 - Geographical distribution of socioeconomic status (SES) and rural conditions, by municipality 
The annual average of age-standardized incidence rates (per 100,000 inhabitants, 95\% confidence intervals) of HF were 210 (207 - 212) for women and 102 (100 - 105 for men (direct method, 2006 European standard population). There is an accentuated geographic pattern, with the highest incidence rates in the northeast, and south of Portugal[6]. Table 2 shows the annual average of age-specific incidence rates of HF per SES and Sex. In general, youngest adults living in areas with higher SES had a lower risk of HF compared to those living in lower SES areas and the reverse occurs for the oldest individuals.

Initially, a main effect model was considered (not including interaction term: $\beta_{12}=0$ ) and adjusting for age and rural conditions, the highest risk of HF was in the deprived SES areas in both sexes. Among women, there was a trend in HF incidence and SES: the risk decreased as the SES increased. Among men, such a trend was not observed (Table 1).

Table 1 - Mean age (95\% Confidence Intervals) at admission, absolute incidence (\%) and Relative Risk of hip fracture ( $95 \%$ Credible Intervals) by sex and socioeconomic status (SES)

Table 2 - Age-specific incidence rates of hip fracture by sex and socioeconomic status (SES)

Considering the model described above, an interaction between age and SES in HF incidence was observed for both sexes: in medium and affluent areas the incidence rates were lower in younger agegroups, and higher in older age-groups, when compared with the most deprived areas (Figure 2 and Table 3). The relative risks (RR) were statistically significant in age groups of 75-79, 80-84 and 85+ in affluent municipalities (using deprived municipalities as reference) in both sexes and in the borderline of the significance in other age groups, such as in women with ages between $50-54$ years (Table 3).

Figure 2 - Relative risk of hip fractures, by sex, age group and three classes of socioeconomic status, using the most deprived as reference

Table 3 - Relative risk of hip fractures $(95 \% \mathrm{Crl})$ by sex, age group and three classes of socioeconomic status, using the most deprived as reference

Figure 3 shows the significant higher and lower RR of HF attributed to unobserved spatial effect. It seems that there are two areas of municipalities with particularly higher risk of HF (Northeast and the very South). This may be related with unobserved variables and future investigation needs to be performed. 
Figure 3 - Significant higher and lower municipality-specific unobserved effect of hip fractures

\section{Discussion}

Our study suggests that, at an ecological level, the main effect of the risk of HF is related with the SES of patients' municipality of residence. In both sexes, a higher risk was observed in deprived municipalities and a trend was identified in women: more affluent areas, lower risk; however some of our results are in the borderline of significance or are not statistically significant.

The inverse relation between HF risk and SES observed in our study is in accordance with a set of studies that explored this issue. In Oslo (Norway) [29] a study including population aged 50 years and over found an higher risk of HF in deprived areas compared with more affluent ones; in Geneva (Switzerland) [30] a study also including population aged 50 years and over found an higher risk in low-income regions compared with medium/higher income regions and their results were similar to a study in Sweden [13] including population in all ages. The same direction of this association was found in an Australian study [16], using a private health insurance as a proxy of higher SES and including population aged 65 years and over. In the Trend region (north of England) [7] a study using the Townsend deprivation index as a proxy of SES, found a significant inverse association in the risk of falls; although, no association between HF and deprivation was found [7] and this may be due to the low statistical power of the study [15] or because only individuals aged $>74$ years were included in the study (the association between SES and HF can be dependent of the age group). In the United States [31], in a community-dwelling of people aged $\geq 70$ years and over there was a non-significant inverse association between HF and income.

Socioeconomic status seem to be also related with bone mineral density - individuals with higher income [32] and higher education [33] had higher BMD when compared with individuals with lower income and education.

The higher risk of HF in individuals living in deprived areas may result from a combination of lifestyle, environmental and social factors: unhealthy lifestyles can lead to higher risk of osteoporosis[2]; inappropriate built environment can lead to higher risk of falls [34]; and lower access to health services can lead to lower access to actions for osteoporosis prevention [35]. Even though in Portugal access to the national health-care system is universal and tendentiously free-of-charge, regions with lower SES can be more isolated, with lower street connections and lack of public transports which may difficult the access to health centers.

The observed increasing trend, at individual level, of the mean age at admission with the decrease in SES can be due to the age structure of the population since the regions with lower SES have the highest proportion of elderly. 
An interaction between SES and age was observed, more accentuated among men: youngest adults living in areas with higher SES had a lower risk of HF compared to those living in lower SES areas and the reverse occurs for the oldest individuals. Few studies have evaluated the age interaction between $\mathrm{HF}$ and SES, but results similar to ours were found in Wales and Denmark $[15,18]$. The lower risk in the youngest living in more affluent areas may be a result of a cohort effect: higher education in the younger may influence risk behaviors [18]. In Wales, among individuals aged < 75 years an inverse association was observed between HF incidence and deprivation of the region of residence, although the relationship disappears in older age groups ( $\geq 75$ years) [15]. In Denmark, the association between HF risk and income was not significant and a possible reason can be the reduced social inequalities [18]. In Spain, an interaction between age and income was found in the relation between BMD and income: youngest adults (20-39 years-old) had a higher BMD in the more affluent areas compared to the more deprived areas and a reverse relation was observed in the older ages [32]. A U-shape relation between BMD and SES was also found in Australia (women, over 18 years) - individuals living in the extreme categories of SES had lower Bone Mineral Density (BMD) [36]

For women, the age interaction with SES was not so clear: older women from areas in the medium SES had lower risk compared to women from lower SES areas and we need further studies to understand if this pattern is related to risk factors such as obesity, physical activity, occupational work, etc; risk for women in the age group 70-74 years was similar in the three SES areas and this may be due to the fact that in Portugal the health guidelines for osteoporosis prevention are mainly focusing in women between 65-74 ages[37].

A limitation of our study is the absence of individual data for SES and for previous places of patient's residence; therefore conclusions need to be taken with caution. The age interaction on the relative risk between HF and SES could be a consequence of changes in place of residence of the elderly. Older and frail individuals, living in more isolated and deprived municipalities, may have moved to more affluent municipalities to live with or close to caregivers. However, other studies tested this hypothesis and nonsignificant differences were found in the mean change of SES on moving to another place [15]. Our study refers to a period of eleven years at nationwide level and it is unlikely that the eventual migration within the country would affect a high percentage of the elders. Because of confidentiality issues we could not identify recurrent fractures and this might be seen as a limitation since the risk of a new fragility fracture increases after the primary hip fracture. However, in our perspective, such limitation does not bias our results since there is no reason to believe that recurrent fractures would be differential among geographic areas or SES groups.

The strength of our study is that we used longitudinal data from nationwide registers and a rigorous study design that minimize the risk of selection and information bias. Besides, we used powerful statistical analysis to measure the association between socioeconomic status and hip fractures. The use of socioeconomic status per municipality was helpful in recognizing areas of higher risk of HF and can help in the design of public health intervention programs. 


\section{Conclusion:}

Ecological studies can help in the identification of areas/population groups at higher risk of hip fractures.

This area-level study suggests a general pattern, the more deprived municipalities presented a higher risk of hip fracture, and an interaction between age group and socioeconomic status of the municipality of residence in the risk of hip fracture; the association between hip fractures incidence and socioeconomic status was inverse in younger ages (lower risk in more affluent areas) and direct in older ages (higher risk in more affluent areas). These results may help decision-makers to better understand the epidemiology of hip fractures and better direct the implementation of political decisions.

\section{References}

1. Morrison A, Fan T, Sen SS, Weisenfluh L. Epidemiology of falls and osteoporotic fractures: a systematic review. ClinicoEconomics and outcomes research : CEOR. 2013;5:9-18. Epub 2013/01/10.

2. NIH Consensus Development Panel on Osteoporosis Prevention, Diagnosis, and Therapy, March 7-29, 2000: highlights of the conference. South Med J. 2001;94(6):56973. Epub 2001/07/07.

3. Ivergård M, Svedbom A, Hernlund E, Compston J, Cooper C, Stenmark J, et al. Epidemiology and Economic Burden of Osteoporosis in Portugal. A report prepared in collaboration with the International Osteoporosis Foundation (IOF) and the European Federation of Pharmaceutical Industry Associations (EFPIA). Archives of osteoporosis. 2013;8:137 (1-2):pag 167.

4. Bischoff HA, Stahelin HB, Dick W, Akos R, Knecht M, Salis C, et al. Effects of vitamin D and calcium supplementation on falls: a randomized controlled trial. J Bone Miner Res. 2003;18(2):343-51. Epub 2003/02/06.

5. Kanis JA, Pitt FA. Epidemiology of osteoporosis. Bone. 1992;13 Suppl 1:S7-15.

6. Pina MF, Alves SM, Barbosa M, Barros H. Hip fractures cluster in space: an epidemiological analysis in Portugal. Osteoporos Int. 2008.

7. West J, Hippisley-Cox J, Coupland CA, Price GM, Groom LM, Kendrick D, et al. Do rates of hospital admission for falls and hip fracture in elderly people vary by socio-economic status? Public Health. 2004;118(8):576-81. Epub 2004/11/09.

8. Kanis JA, Borgstrom F, Compston J, Dreinhofer K, Nolte E, Jonsson L, et al. SCOPE: a scorecard for osteoporosis in Europe. Archives of osteoporosis. 2013;8(12):144. Epub 2013/09/14.

9. Benetos IS, Babis GC, Zoubos AB, Benetou V, Soucacos PN. Factors affecting the risk of hip fractures. Injury. 2007;38(7):735-44. Epub 2007/02/20.

10. Korpelainen R, Korpelainen J, Heikkinen J, Vaananen K, KeinanenKiukaanniemi S. Lifelong risk factors for osteoporosis and fractures in elderly women with low body mass index--a population-based study. Bone. 2006;39(2):385-91. Epub 2006/03/15.

11. Farahmand BY, Persson PG, Michaelsson K, Baron JA, Parker MG, Ljunghall $\mathrm{S}$, et al. Socioeconomic status, marital status and hip fracture risk: a population-based case-control study. Osteoporos Int. 2000;11(9):803-8. Epub 2001/01/10.

12. Icks A, Haastert B, Wildner M, Becker C, Rapp K, Dragano N, et al. Hip fractures and area level socioeconomic conditions: a population-based study. BMC Public Health. 2009;9:114. Epub 2009/04/29. 
13. Farahmand BY, Persson PG, Michaelsson K, Baron JA, Parker MG, Ljunghall S. Socioeconomic status, marital status and hip fracture risk: a population-based casecontrol study. Osteoporos Int. 2000;11(9):803-8. Epub 2001/01/10.

14. Suen LK. Occupation and risk of hip fracture. J Public Health Med. 1998;20(4):428-33. Epub 1999/01/29.

15. Jones S, Johansen A, Brennan J, Butler J, Lyons RA. The effect of socioeconomic deprivation on fracture incidence in the United Kingdom. Osteoporos Int. 2004;15(7):520-4. Epub 2004/04/02.

16. Peel NM, McClure RJ, Hendrikz JK. Psychosocial factors associated with fallrelated hip fractures. Age Ageing. 2007;36(2):145-51. Epub 2007/01/30.

17. Brennan SL, Pasco JA, Urquhart DM, Oldenburg B, Hanna F, Wluka AE. The association between socioeconomic status and osteoporotic fracture in population-based adults: a systematic review. Osteoporos Int. 2009;20(9):1487-97. Epub 2008/12/25.

18. Vestergaard P, Rejnmark L, Mosekilde L. Socioeconomic aspects of fractures within universal public healthcare: a nationwide case-control study from Denmark. Scandinavian journal of public health. 2006;34(4):371-7. Epub 2006/07/25.

19. Reimers A, Laflamme L. Hip fractures among the elderly: personal and contextual social factors that matter. J Trauma. 2007;62(2):365-9. Epub 2007/02/14.

20. INE. Instituto Nacional de Estatística; [cited 2012]; Available from: www.ine.pt.

21. OECD. Divided we stand: why inequality keeps rising. An Overview of Growing Income Inequalities in OECD Countries: Main Findings. 2011; Available from: http://www.oecd.org/social/inequality.htm.

22. Barros PP, Machado SR, Simoes Jde A. Portugal. Health system review. Health systems in transition. 2011;13(4):1-156. Epub 2012/01/10.

23. ACSS. Auditoria da codificação clínica [Clinical codification Audits]. 2011 Available from: http://portalcodgdh.minsaude.pt/index.php/Auditoria_da_codificação_cl\%C3\%ADnica.

24. Moreira J. Fracturas Osteoporóticas do Colo do Fémur em Portugal e seus Determinantes Socioeconómicos. Porto: Faculdade de Engenharia da Universidade do Porto.; 2008.

25. Chevalley T, Herrmann FR, Delmi M, Stern R, Hoffmeyer P, Rapin CH, et al. Evaluation of the age-adjusted incidence of hip fractures between urban and rural areas: the difference is not related to the prevalence of institutions for the elderly. Osteoporosis international : a journal established as result of cooperation between the European Foundation for Osteoporosis and the National Osteoporosis Foundation of the USA. 2002;13(2):113-8. Epub 2002/03/22.

26. Banerjee S, Carlin BP, Gelfand AE. Hierarchical modeling and analysis for spatial data. In: Hall/CRC Ca, editor.2004.

27. R Core Team. R: A language and environment for statistical computing. $\mathrm{R}$ Foundation for Statistical Computing. Vienna, Austria2012.

28. Spiegelhalter D, Thomas A, Best N, Lunn D. WinBUGS User Manual (Version 1.4). 2003 January 2003. Report No.

29. Kaastad TS, Meyer HE, Falch JA. Incidence of hip fracture in Oslo, Norway: differences within the city. Bone. 1998;22(2):175-8. Epub 1998/02/26.

30. Guilley E, Herrmann F, Rapin CH, Hoffmeyer P, Rizzoli R, Chevalley T. Socioeconomic and living conditions are determinants of hip fracture incidence and age occurrence among community-dwelling elderly. Osteoporos Int. 2011;22(2):647-53. Epub 2010/05/19. 
31. Wilson RT, Chase GA, Chrischilles EA, Wallace RB. Hip fracture risk among community-dwelling elderly people in the United States: a prospective study of physical, cognitive, and socioeconomic indicators. Am J Public Health. 2006;96(7):1210-8. Epub 2006/06/01.

32. del Rio Barquero L, Romera Baures M, Pavia Segura J, Setoain Quinquer J, Serra Majem L, Garces Ruiz P, et al. Bone mineral density in two different socioeconomic population groups. Bone Miner. 1992;18(2):159-68. Epub 1992/08/01.

33. Varenna M, Binelli L, Zucchi F, Ghiringhelli D, Gallazzi M, Sinigaglia L. Prevalence of osteoporosis by educational level in a cohort of postmenopausal women. Osteoporos Int. 1999;9(3):236-41. Epub 1999/08/18.

34. Rapiti E, Fioretta G, Schaffar R, Neyroud-Caspar I, Verkooijen HM, Schmidlin $\mathrm{F}$, et al. Impact of socioeconomic status on prostate cancer diagnosis, treatment, and prognosis. Cancer. 2009;115(23):5556-65. Epub 2009/09/30.

35. Neuner JM, Zhang X, Sparapani R, Laud PW, Nattinger AB. Racial and socioeconomic disparities in bone density testing before and after hip fracture. $\mathrm{J}$ Gen Intern Med. 2007;22(9):1239-45. Epub 2007/06/28.

36. Brennan SL, Henry MJ, Wluka AE, Nicholson GC, Kotowicz MA, Williams $\mathrm{JW}$, et al. BMD in population-based adult women is associated with socioeconomic status. J Bone Miner Res. 2009;24(5):809-15. Epub 2008/12/31.

37. Alves SM, Economou T, Oliveira C, Ribeiro AI, Neves N, Gomez-Barrena E, et al. Osteoporotic hip fractures: Bisphosphonates sales and observed turning point in trend. A population-based retrospective study. Bone. 2012. Epub 2013/01/01. 